\title{
ComparaçÃo de ReSULTAdo ENTRE AS EQUAÇões DE COMPOSIÇÃO CORPORAL DE JACKSON \& Pollock de três e sete dobras cutâneas
}

Tarcísio Margoti' tmargoti@bol.com.br

doi:10.3900/fpi.8.3.191.p

Margoti T. Comparação de resultado entre as equações de composição corporal de Jackson \& Pollock de três e sete dobras cutâneas. Fit Perf J. 2009 mai-jun;8(3):191-8.

\section{RESUMO}

Introdução: $\bigcirc$ propósito desse estudo foi comparar os resultados obtidos através das equações de estimativa de percentual de gordura (\%G) de Jackson \& Pollock de três dobras cutâneas (3DC) e sete dobras cutâneas (7DC). Materiais e Métodos: A amostra foi composta por um grupo de 51 indivíduos, 30 do gênero masculino e 21 do gênero feminino, com idade entre 18 e 47 anos, sendo estes acadêmicos do curso de Educação Física da Universidade do Contestado, Campus Universitário de Curitibanos - SC, que foram selecionados aleatoriamente. Estes foram avaliados uma única vez, onde os valores coletados foram idade e espessura das dobras cutâneas tricipital, subescapular, peitoral, axilar média, suprailíaca, abdominal, e coxa, tendo as 7DC sido aferidas três vezes cada, utilizando-se o valor médio. Os resultados foram lançados nas equações, utilizando-se o programa Microsoft Excel ${ }^{\circledR}$ para a obtenção do percentual de gordura, posteriormente comparados e analisados estatisticamente com o Teste Z. Resultados: Quando comparadas as duas equações, pôde-se observar que houve uma variação de 0,83\% para o gênero masculino entre as duas equações, e 1,97\% para o gênero feminino. Outro fator analisado durante a comparação das mesmas equações, mas agora em relação ao gênero, é que 56,7\% da amostra masculina apresentou valor inferior de \%G na equação de 7DC, o que se inverte no gênero feminino, onde 90,5\% apresentou valor superior na equação de 7DC. Essas diferenças de resultado são significativas quando comparados estatisticamente. Discussão: Isso mostra que a diferença entre os resultados das equações é pequena e, estatisticamente não significativa. E que, conforme a quantidade e local de DC utilizadas, e também as características de cada gênero, fazem com que existam estas diferenças.

\section{PALAVRAS-CHAVE}

Tecido Adiposo Marrom, Tecido Adiposo Branco, Pregas Cutâneas.

1 Universidade do Contestado - UnC - Curitibanos - Brasil

Copyright(C 2009 por Colégio Brasileiro de Atividade Física, Saúde e Esporte

Fit Perf J | Rio de Janeiro | 8 | 3 | 191-198 | mai/jun 2009 


\section{Comparison of results between the equations of body composition of Jackson \& Pollock of three and SEVEN SKINFOLDS}

\section{ABSTRACT}

Introduction: This research has a principal intention, that compare the results obtained through equations of estimate fat percentage (\% $\mathrm{G})$ by Jackson \& Pollock of the three skinfolds (3SF) and seven skinfolds (7SF). Materials and Methods: The sample was made up of a group of the 51 subjects, they been 30 of masculine gender and 21 of feminine gender, they had age among 18 until 47 years old, these academics of Physical Education course of Contestado University - SC - Brazil, they went random selects. They went values a only time, where the collect's values went the age and the thickness of the skinfolds triciptal, subescapular, bosom, middlearmpit, supra iliaca, abdominal and thigh, having inspect three times each, using itself of middle of each site. Which were released at the equations by using the Microsoft Excel ${ }^{\circledR}$ program to obtain of the fat percentage afterwards compared and statistically analyzed with the Test Z. Results: When it compared the two equations, to be possible book that there was a variation of $0.83 \%$ to masculine gender between two equations, and $1.97 \%$ to feminine gender. Another analyzed factor during the confrontation of the same equations, but now in respect to gender, is that $56.7 \%$ of the masculine sample it presented inferior value of the $\% \mathrm{G}$ in the equation $7 S F$, that change itself in the feminine gender where $90.5 \%$ presented superior value in the equation of 7 SF. These are significant differences in outcome when compared statistically. Discussion: This study shows that the difference among the results of the equations is small and it no statistically significant. And that, accordant the quantity and local of skinfolds used, and also the characteristics of each gender, they do that there were these differences.

\section{KEYWORDS}

Adipose Tissue, Brown, Adipose Tissue, White, Skinfold Thickness.

\section{Comparación de resultados entre las ecuaciones de la composición corporal de Jackson \& Pollock de TRES Y SIETE PLIEGUES CUtÁNEOS}

\section{RESUMEN}

Introducción: El propósito de este estudio fue comparar los resultados obtenidos mediante las ecuaciones de estimación del porcentaje de grasa $(\% \mathrm{G})$ y Jackson Pollock de tres pliegues cutáneos (3PC) y siete pliegues cutáneos (7PC). Materiales y Métodos: La muestra comprende un grupo de 51 personas, 30 hombres y 21 mujeres, de edades comprendidas entre los 18 y 47 años, estos curso académico de la Educación Física, Universidad de Contestado - SC - Brasil, que fueron seleccionados al azar. Estas fueron examinadas una vez, cuando se recogieron los datos para los siguientes años y el espesor del pliegue cutáneo de tríceps, subescapular, pectoral, axilar medio, supra ilíaco, abdominal, muslo y, con 7PC marcada, tres veces cada una, utilizando el promedio de cada sitio. Que se pusieron en marcha en las ecuaciones utilizando el programa Microsoft Exce ${ }^{\circledR}$ para obtener el porcentaje de grasa y, a continuación, comparar y analizar estadísticamente con la prueba Z. Resultados: Comparando las dos ecuaciones, se observó que hubo una variación de 0,83\% para los hombres entre las dos ecuaciones, y 1,97\% para las mujeres. Otro factor considerado en la comparación de estas ecuaciones, pero ahora en relación con el género, es que el 56,7\% de la muestra masculina mostró menor valor de \%G en la ecuación de 7PC, lo que se invierte en las mujeres, dónde 90,5\% mostró un mayor valor en la ecuación de 7PC. Estas diferencias en los resultados son estadísticamente significativos en comparación. Discusión: Esto demuestra que las diferencias entre los resultados de las ecuaciones es pequeña y no estadísticamente significativa. Y que la cantidad y la ubicación de pliegue cutáneo utilizado, y también las características de cada género, hacen que estas diferencias existen.

\section{PALABRAS CLAVE}

Tejido Adiposo Pardo, Tejido Adiposo Blanco, Grosor de Pliegues Cutáneos.

\section{INTRODUÇÃO}

No Brasil a questão estética corporal é muito valorizada, fato este que induz a população a acompanhar e controlar o seu peso. E neste processo para manter um peso saudável, o percentual de gordura (\%G) é um dos fatores que podem influenciar na alteração do mesmo, onde uma pessoa obesa na sociedade brasileira não é vista como tendo uma estética agradável. Além do mais, este alto \%G apresentado por um obeso pode resultar em prejuízos para a saúde.

Os profissionais da área da saúde, como médicos e nutricionistas, entre outros, podem direcionar esta população para a melhor forma de controlar o estado de seu corpo, estando estes também cientes de como se encontram fisicamente, pois, além de contribuírem cientificamente, também estão constantemente sendo observados. Você frequentaria um médico cardiologista ou um nutricionista que fosse obeso, sabendo que a obesidade é um grande fator de risco e que um nutricionista trabalha, principalmente, no controle do peso e cobra isso de seus clientes? Claro que não!

Ressalte-se também que qualquer um destes tem capacidades profissionais para controlar o estado físico de seu próprio corpo de maneira simples. No caso 
deste excesso não ser resolvido através de dietas e exercícios físicos, devidos a hereditariedades genéticas, estes profissionais deveriam ser capazes de procurar auxílio com outros métodos de tratamento. Desta forma estariam auxiliando não somente com a orientação de tratamento de seus pacientes, mas também com o exemplo.

Isso pode ser visto também nos profissionais da área da atividade física, pois os mesmos ensinam que se deve ter um corpo saudável, com o peso ideal. No entanto, em que estado se encontra o corpo desses?

Pensando desta maneira que se buscou avaliar a composição corporal destes profissionais, já que vive-se em um mundo onde se busca sempre alguém para se espelhar, seja em que área for. Torna-se utópico querer afirmar que se deve exercitar para manter o corpo em forma, mas não o faz.

Um cuidado deve ser tomado quando se refere à estética corporal e ao corpo saudável, pois somente a estética não é o suficiente para se concluir que uma pessoa é obesa ou não. Podem existir variações na composição do corpo humano, como o \%G e a massa corporal magra - tecidos isentos de gordura, como músculos, ossos e resíduos ${ }^{1}$-, que alteram a estética, mas não necessariamente torna a pessoa obesa. Desta forma, uma pessoa robusta, pode não ser obesa, pois o seu corpo apresentará uma grande quantidade de massa magra, assim como uma pessoa com uma agradável estética pode ter seu \%G muito alto.

Como em se tratando de avaliação física existem várias equações preditivas (fórmulas para avaliação), cada qual com seu objetivo, e estando este estudo voltado para a avaliação do percentual de gordura ao longo do corpo, foram utilizadas as equações de Jackson \& Pollock de 3DC e 7DC.

\section{Objetivo geral}

Verificar se existe diferença nos resultados da composição corporal de um mesmo avaliado, utilizando-se as equações de avaliação física de Jackson \& Pollock de 3DC e 7DC entre os acadêmicos do curso de Educação Física da Universidade do Contestado - Campus Curitibanos - SC.

\section{Objetivos específicos}

- Apresentar um embasamento teórico sobre o processo de aferição das medidas e das equações utilizados;

- Coletar valores referentes a dobras cutâneas;

- Definir a variação de percentual de gordura entre as duas equações;

- Definir a variação de percentual de gordura entre as equações, com relação ao gênero; $e$
- Verificar se os acadêmicos estão com excesso de massa gorda.

\section{MATERIAIS E MÉTODOS}

Alguns procedimentos foram estabelecidos e seguidos. Iniciou-se com uma investigação realizada através de pesquisa bibliográfica, resultando em uma revisão de literatura (basicamente qualitativa). Em seguida foi realizada uma pesquisa de campo na Universidade do Contestado - UnC - Campus Universitário Curitibanos - SC, com os 150 acadêmicos do curso de Educação Física, dos quais foram sorteados $30 \%$, ou seja, 51 acadêmicos que serviram de amostra, sendo 30 homens e 21 mulheres, com idade entre 18 e 61 anos.

A pesquisa consiste na avaliação do $G \%$ - porção quantitativa do estudo - desta amostra, na qual foi utilizada para obtenção de valores o método de dobras cutâneas, que apresenta locais pré determinados para sua aferição: região subescapular; peitoral; axilar média; abdominal; coxa; tríceps; e suprailíaca. Estas medidas foram aferidas três vezes, sendo que foram aferidas todas as regiões uma primeira vez, repetindo o processo inteiro mais duas vezes. Assim, chegou-se a uma média de cada local de aferição, servindo esta de base para avaliar o percentual de gordura.

Esta aferição foi realizada com um instrumento chamado de plicômetro, também conhecido como compasso, da marca Lange, que apresenta uma precisão de $1,0 \mathrm{~mm}$ e uma pressão constante de $10 \mathrm{~g} \cdot \mathrm{mm}^{-2}$. A aferição procedeu-se no pinçamento das dobras citadas anteriormente, com o dedo indicador e com o polegar longitudinalmente ao músculo - cuidado deve ser tomado para não se pinçar o músculo -, onde se introduz as pinças do plicômetro e verifica o valor apresentado no mostrador do aparelho.

Após a obtenção dos valores, estes foram lançados nas equações, em uma planilha eletrônica, sendo usados para chegar ao resultado final, ou seja, ao percentual de gordura. A partir daí foi calculada a média de variação entre os resultados dos homens e das mulheres e, posteriormente, o comparativo entre os resultados apresentados pelas duas equações. Assim, verificou-se se havia diferença significativa entre os resultados das duas equações.

Os cálculos citados foram realizados e tabulados no programa Microsoft Excel ${ }^{\circledR}$, sendo utilizado o Teste Z para análise estatística.

\section{Composição corporal}

A composição corporal é tudo aquilo que faz parte do corpo humano, ou seja, a pele, os músculos, os ossos, a massa gorda (gordura) e os órgãos, totali- 
zando o peso corporal total. Segundo Pitanga ${ }^{2}$, "... os cientistas, para fins didáticos, dividem o corpo em quatro componentes. $\bigcirc$ peso corporal, então, passa a ser igual à soma da massa de gordura, da massa óssea, da massa muscular e da massa residual, sendo que no último componente estão incluídos os diversos órgãos do corpo e a pele".

É da composição corporal que é aferido o \%G. Podendo ser este regional - de uma parte do corpo, como o tronco ou membro superior, por exemplo - ou total - utilizando amostras do corpo todo, o que trará resultados mais garantidos, pois, dadas as diferenças entre os membros superiores e inferiores, esta se utiliza de valores de ambos os locais. É preciso estar ciente de que estes critérios podem variar conforme a cor, raça, idade, sexo, etc.

\section{Medidas e avaliação física}

Técnica pela qual se consegue observar o estado físico de uma pessoa, este se tornando tanto qualitativo como quantitativo, pois, a partir do momento em que se refere à medida, esta se depara com a determinação de uma grandeza, o que, posteriormente, levará a uma avaliação qualitativa baseado nos resultados obtidos. A avaliação é um processo de tomada de decisões que estabelece um julgamento de valores sobre a qualidade de algo que se tenha medido ${ }^{2}$.

A avaliação deve respeitar uma metodologia onde se utiliza um processo preciso - o quê, como, por quê, etc. -, com validade, e, assim, fidedigno, e que também apresente objetivos concretos - metodologia esta que será explicada posteriormente - e assim, exprimam os resultados esperados, dando então valor real à informação coletada.

Vários são os fatores que estão levando as pessoas a realizarem uma avaliação física, principalmente reforçada com a ideia atual de corpo saudável (boa estética corporal), pois, para chegar a este estado, é necessária a realização de alguns tipos de atividade física e, certamente, se este for bem acompanhado por um profissional responsável, será direcionada à execução da avaliação, passando pelos três tipos de avaliação citados anteriormente ${ }^{3}$.

Estes resultados, encontrados com a realização da avaliação física, podem ser aplicados para monitorar: os níveis de gordura corporal; quais os riscos que estas alterações podem provocar, desta forma servindo de base para estudos; riscos como as doenças relacionadas à obesidade; entre outros fatores ${ }^{4,12}$. Portanto, isto leva à ideia de que, para a verificação do estado de um corpo, a avaliação é muito útil e recomendada e pode ser feita a qualquer momento, desde que acompanhada por um profissional, o que, além de transmitir segurança ao avaliado, também traz fidedignidade ao resultado ${ }^{\mathbf{5}}$. Estes fatores estão diretamente relacionados ao \%G e servem de alerta para a tomada de precauções na rotina diária, como é o caso do risco cardiovascular.

Todos estes fatores refletem em ganhos para a população, pois estarão sendo alertados de como estão vivendo, e reflete positivamente também para os profissionais responsáveis por estas avaliações, dado a procura frequente do mesmo. No entanto, cabe aos profissionais tomarem consciência do como estão fisicamente, a fim de manter uma boa imagem, pois, se estiverem acima do peso serão vistos como não conhecedores do assunto, podendo provocar uma desmotivação dos clientes, que serão levados a pensar: "se o próprio avaliador não apresenta um porte físico bom, certamente o método que ele apresenta não é bom".

\section{Método de avaliação por dobras cutâneas}

Conforme Heyward \& Stolarzyk ${ }^{4}$, a dobra cutânea é uma medida da espessura de duas camadas de pele e a gordura subcutânea adjacente. Estas gorduras subcutâneas, dispostas por todo o corpo de forma irregular, correspondem a um terço da gordura corporal total, segundo Lohman apud Heyward \& Stalarzyk ${ }^{4}$, pois ainda encontram-se gorduras intramusculares, intermusculares e dentro dos órgãos internos. No entanto, Tritschler ${ }^{6}$ afirma que este valor está estimado em duas metades, uma subcutânea e a outra interna. Porém, a avaliação da gordura subcutânea serve de base e é a mais usada atualmente para calcular a estimativa de gordura corporal, devido a alguns fatores que Pitanga ${ }^{2}$ menciona.

Com relação à antropometria, a técnica de espessura do tecido adiposo subcutâneo parece ser a mais adequada para estudos da composição corporal em nosso meio, considerando principalmente o menor custo dos aparelhos utilizados, a não-invasividade do método, a rapidez e a facilidade para interpretação dos resultados, bem como a boa correlação entre as medidas antropométricas e a densidade corporal.

A variação na distribuição da gordura está relacionada a fatores como sexo, idade e grau de obesidade, sendo que as equações que são usadas para estas estimativas respeitam estes fatores e variam conforme a população. Esta variação pode ser observada também conforme o local do corpo, e que, conforme fatores genéticos e ambientais, pode-se encontrar mais massa gorda na região do tronco superior e menos na região inferior em algumas pessoas e, em outras, o oposto ${ }^{7}$. 
Quando se refere ao gênero, existe uma variação no \%G de homens e mulheres, onde as mulheres possuem um índice superior aos homens ${ }^{6}$.

Atualmente existem vários locais de aferição das dobras cutâneas ${ }^{2}$, mas devido à necessidade de se pinçar somente gordura e pele, deixando de fora a composição muscular, este número diminui bastante.

Pitanga $^{\mathbf{2}}$ e Fernandes Filho ${ }^{\mathbf{7}}$, entre outros autores, citam os seguintes locais e dobras cutâneas como sendo as principais: triciptal, subescapular, peitoral, axilar média, suprailíaca, abdominal, e coxa. A Figura 1 mostra uma das dobras cutâneas avaliadas, o peitoral.

$O$ processo de pinçamento das DC deve ser feito com o dedo indicador e com o polegar, sempre no lado direito do avaliado, com o compasso (adipômetro) entrando perpendicular à dobra, esperar de $2 \mathrm{~s}$ a $4 \mathrm{~s}$ - existindo variações deste tempo, conforme o autor - para realizar a leitura; a pele do avaliado deve estar seca, sem óleo, para que o pinçamento seja possível, ou seja, não escape da mão ou do compasso ao ser aferida; não deve ser aferida sobre a roupa, pois terá sua exatidão alterada.

Cada uma deve ser aferida de duas a três vezes e em circuito, ou seja, não aferir a mesma dobra as três vezes consecutivas, pois ao ser aplicada pressão na dobra a gordura subcutânea tende a se comprimir e, assim, diminuir o valor. Recomenda-se aferir todas as dobras uma vez e, após aferir a última, iniciar novamente pela primeira que foi aferida. Se o valor entre as medidas variar acima de $1 \mathrm{~mm}$ deve-se realizar um novo circuito, onde o valor final será a média entre a média do primeiro com o segundo circuito $\mathbf{1 , 2 , 4 , 7}$.

Não se recomenda usar o método de DC para clientes obesos, devido à possibilidade do diâmetro da dobra poder ultrapassar a capacidade de abertura do compasso e, assim, não ser pinçada totalmente, ou então escapar durante o processo de aferição, o que pode causar desconforto tanto para o avaliado como para o avaliador ${ }^{\mathbf{5}}$.

Figura 1 - (a) Localização da dobra peitoral para homens; (b) Medida da dobra

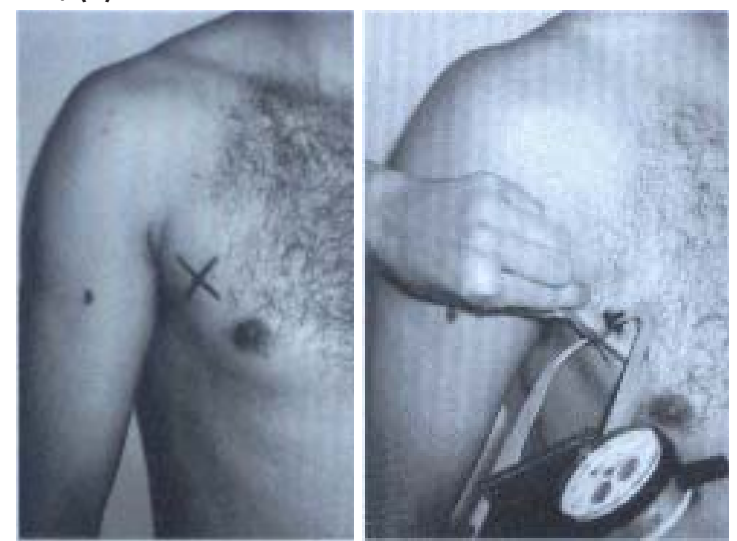

fonte: Queiroga ${ }^{1}$
A questão ética também está relacionada à avaliação física, seja ela da forma que for. Durante este processo são encontrados valores individuais referentes a cada um, os quais são de interesse do mesmo, e também devido à necessidade de procedimentos que requerem confiança e sigilo.

Para executar a avaliação utilizando-se este método necessita-se de um compasso, também conhecido como espessímetro, adipômetro ou plicômetro. Atualmente existem várias marcas de compasso, entre eles o Lange ${ }^{\circledR}$, - Harpenden ${ }^{\circledR}$, o Cescorf ${ }^{\circledR}$, entre outros, sendo que os mais usados são os dois primeiros, devido à sua maior fidedignidade e exatidão na medida.

Estes compassos exercem sobre a dobra uma pressão constante de 10 g. $\mathrm{mm}^{-2}$, ou seja, $10 \mathrm{~g}$ para cada milímetro quadrado da superfície da mandíbula do compasso. Durante a abertura da pinça, entre $2 \mathrm{~mm}$ e $40 \mathrm{~mm}$, não pode ocorrer nesta pressão uma variação superior a $2 \mathrm{~g} \cdot \mathrm{mm}^{-2}$. Se a pressão for muito forte, ocorrerá a compressão da gordura subcutânea e, assim, a diminuição no valor obtido'.

Assim como os aparelhos, existem várias equações para estimar o percentual de gordura corporal. Esta seleção deve ser feita tendo por base os objetivos da pesquisa, pois as equações variam de acordo com o sexo, a idade, a raça, o nível de aptidão física e a gordura corporal da amostra.

Para o calculo do \%G neste método, necessita-se inicialmente, em algumas equações, calcular a densidade corporal (DENS). Após este cálculo, utiliza-se a fórmula de Siri 9 para chegar ao \%G:

$\% G=\left[\left(4,95 *\right.\right.$ DENS $\left.\left.^{-1}\right)-4,50\right] * 100$

Equação de Jackson \& Pollock (1978) usando 7DC DENS para homens:

DENS $=1,12000000-[0,00043499 *(\Sigma 7 D C)]$ $+\left[0,00000055^{*}(\Sigma 7 D C)^{2}\right]$ $[0,00028826 *($ idade $)]$

DENS para mulheres:

DENS $=1,09700000-\left[0,00046971^{*}(\Sigma 7 D C)\right]$ $+\left[0,00000056^{*}(\Sigma 7 D C)^{2}\right]$ $\left[0,00012828^{*}\right.$ (idade)]

Equação de Jackson \& Pollock (1978 e 1980) usando 3DC (PT, AB, CX para homens, e TR, SI, CX para mulheres) DENS para homens:

DENS $=1,1093800-0,0008267^{*}(X 1)+$ $0,0000016^{*}(X 1)^{2}-0,0002574^{*}(X 3)$

DENS para mulheres:

DENS $=1,0994921-0,0009929 *(\times 2)+$ $0,0000023 *(X 2)^{2}-0,0001392 *(X 3)$

Onde:

$\mathrm{X} 1=\Sigma \mathrm{DC}$ peitoral, abdominal e coxa

X2 $=\Sigma D C$ tríceps, supra-ilíaca e coxa

X3 = idade em anos 
Realizado o procedimento de pinçamento das dobras e lançados estes valores nas equações, os resultados obtidos devem ser submetidos à comparação com os padrões estabelecidos - considerando o objetivo do estudo.

\section{Obesidade}

Tristschler ${ }^{6}$ afirma que a obesidade é o nível de excesso de gordura no qual os riscos de saúde relacionados à gordura começam a aumentar.

Nahas $^{10}$ relata alguns destes riscos mencionados por Tritschler como sendo implicações para a saúde. Entre eles estão a maior incidência de doenças cardíacas, a morte prematura e a elevação dos diabetes, entre outras. Portanto, uma pessoa é considerada obesa quando, depois de avaliada, seu \%G é superior aos índices recomendados e começam a surgir sintomas de doenças associadas à gordura corporal ${ }^{6,10}$.

\section{RESULTADOS}

Após realização da coleta de dados entre os acadêmicos do curso de Educação Física da Universidade do Contestado - Campus Universitário de

Gráfico 1 - Comparação entre os valores de percentual de gordura $(\% \mathrm{G})$, obtidos através das equações de Jackson \& Pollock de três dobras cutâneas (3DC) e sete dobras cutâneas (7DC), para o gênero masculino

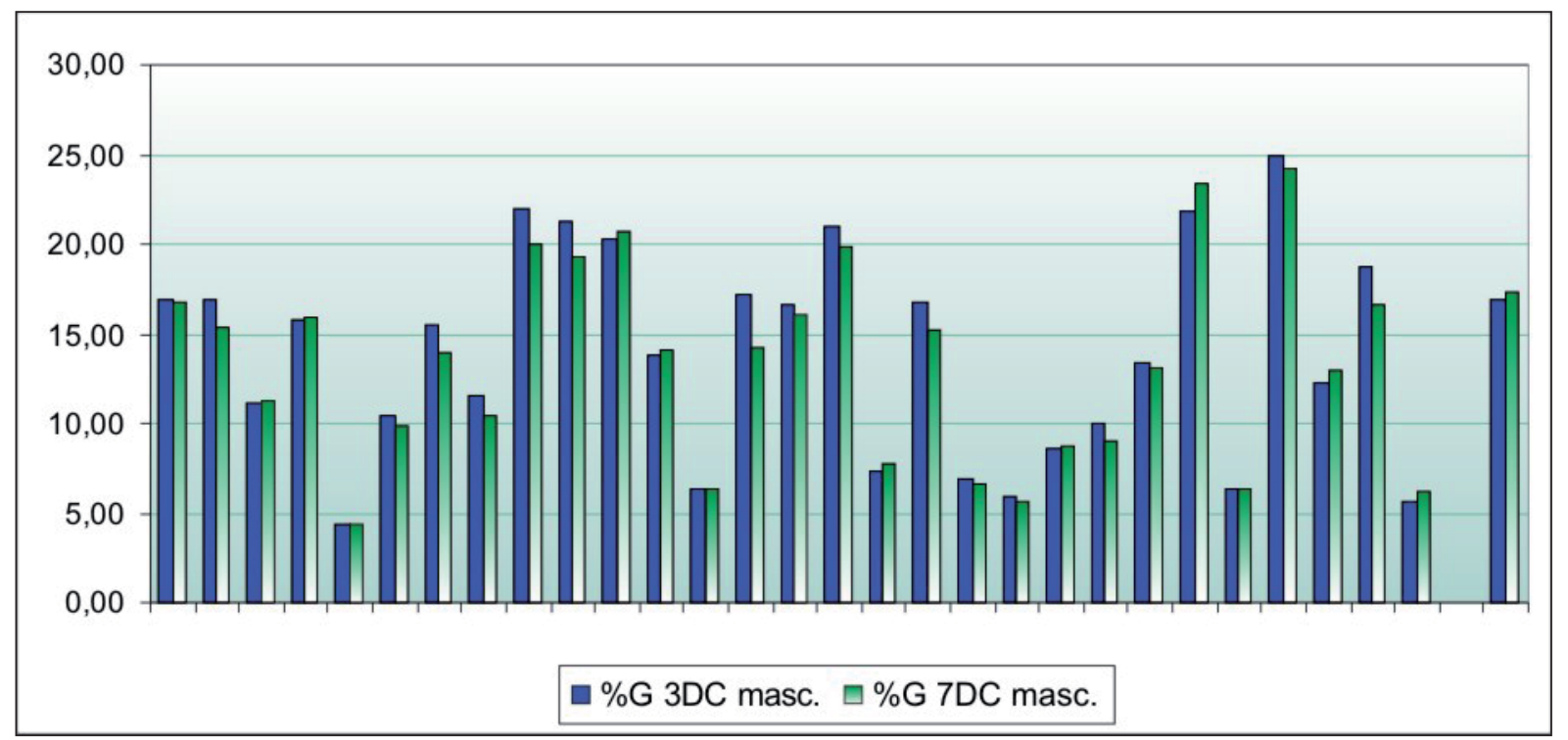

Gráfico 2 - Comparação entre os valores de percentual de gordura $(\% \mathrm{G})$, obtidos através das equações de Jackson \& Pollock de três dobras cutâneas (3DC) e sete dobras cutâneas (7DC), para o gênero feminino

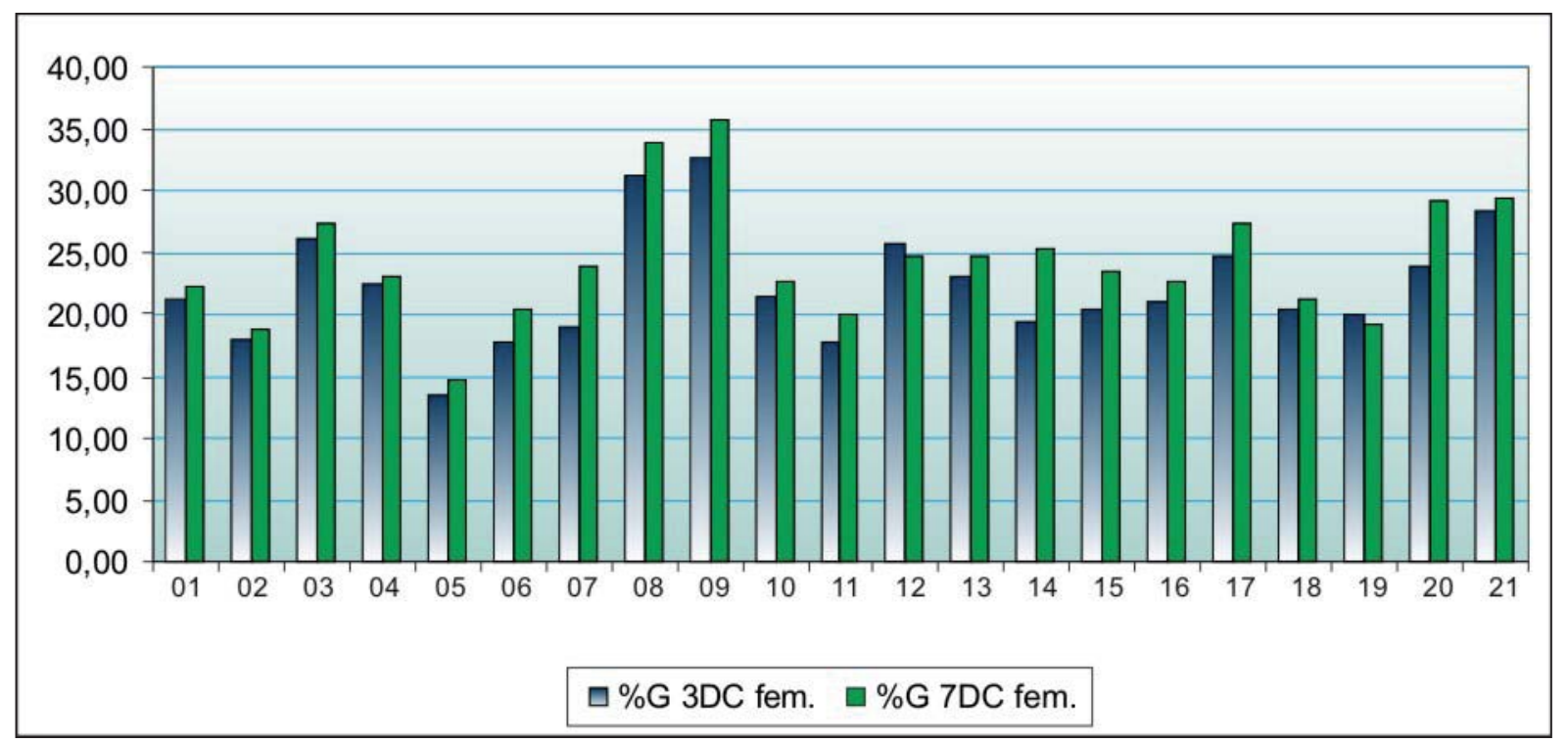


Gráfico 3 - Comparação entre os valores médios de percentual de gordura (\%G) obtido através das equações de Jackson \& Pollock de três dobras cutâneas (3DC) e sete dobras cutâneas (7DC) para os gêneros feminino e masculino

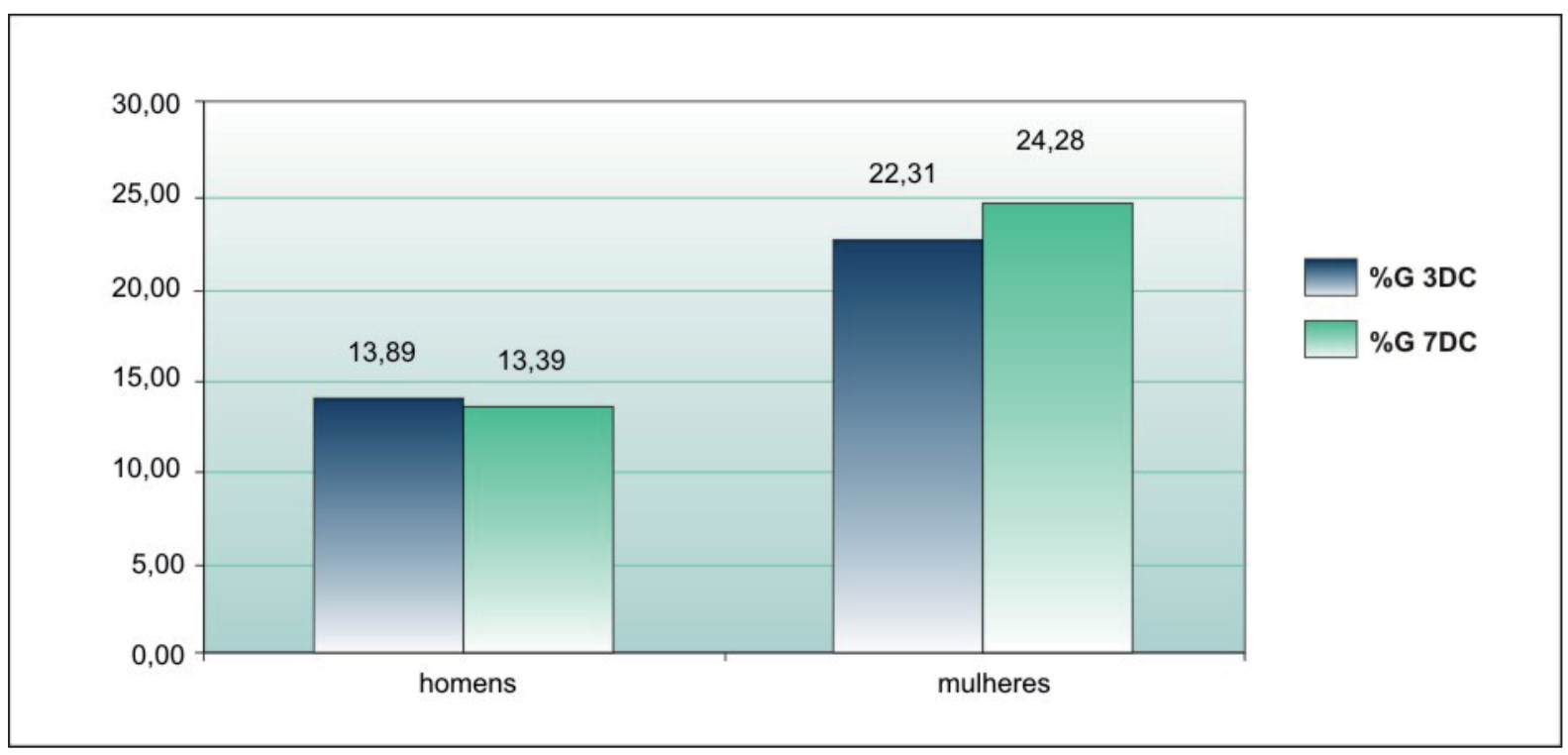

Curitibanos, foi realizada a classificação da amostra segundo tabelas normativas, chegando aos resultados a seguir.

Tem-se na amostra uma população do gênero masculino com média de idade de 23,17 anos, enquanto as mulheres apresentam média de idade de 25,10 anos.

Conforme o Gráfico 1, 56,7\% da amostra masculina apresentou menor valor na equação de Jackson \& Pollock com 7DC do que com 3DC.

Já para o gênero feminino, a maior parte da amostra $(90,5 \%)$ apresentou valor superior na equação de Jackson \& Pollock com 7 DC do que com 3DC (Gráfico 2).

Ao comparar os gêneros tendo por base a mesma equação, pode-se confirmar que as mulheres apresentam um \% G maior que o dos homens em ambas as equações (Gráfico 3).

Já durante a classificação, na comparação entre gêneros e na equação de 3DC, $66 \%$ da amostra feminina e $57 \%$ da amostra masculina não apresentaram excesso de gordura, o que mostra que as mulheres estão mais bem classificadas nesta equação, ou seja, há mais mulheres sem excesso de gordura do que homens, na equação de 3DC. Ainda por esta equação, $24 \%$ das mulheres e $33 \%$ dos homens apresentaram excesso de gordura.

No caso da equação de 7DC, $47 \%$ da amostra feminina e $63 \%$ da amostra masculina não apresentaram excesso de gordura, o que mostra que os homens estão mais bem classificadas nesta equação, ou seja, há mais homens sem excesso de gordura do que mulheres, na equação de 7DC. Ainda por esta equação, $24 \%$ das mulheres e $20 \%$ dos homens apresenta excesso de gordura.
Ao comparar o resultado do $\% G$ entre as duas equações usadas neste estudo, houve uma variação de 0,83\% para o gênero masculino, e 1,97\% para o gênero feminino.

\section{DISCUSSÃO}

O resultado apresentado no Gráfico 1 pode ser causado pela quantidade de DC pinçadas e usadas na equação, pois na equação de 7DC utilizam-se DC de vários locais do corpo (tricipital, subescapular, peitoral, axilar média, supra ilíaca, abdominal e coxa), onde são pinçados locais de grande concentração de gordura (região abdominal, no caso dos homens) e em locais onde a concentração é baixa (em comparação com as outras dobras). Esta diferença na concentração de gordura nos vários locais de aferição pode ser observada também no estudo de Costa ${ }^{8}$, onde verificou-se o valor de cada DC.

Podemos afirmar, com 99\% de confiança, que a diferença existente nos resultados dos Testes de Jackson \& Pollock de 3DC e 7DC entre a amostra masculina não é significativa, conforme resultado do Teste $Z^{11}(Z=0,34)$. Isso significa que pode-se usar qualquer um dos testes, pois em ambos o resultado é fidedigno.

Como foi mencionado no caso dos homens, conforme a população a ser avaliada os resultados podem variar e, assim, apresentar um resultado totalmente diferente dos já estudados, o que aconteceu com a amostra feminina (Gráfico 2). Neste caso, foi encontrado um valor superior do $\% \mathrm{G}$ na equação com 7DC. 
Podemos afirmar também, com 99\% de confiança, que a diferença existente nos resultados dos Testes de Jackson \& Pollock de 3DC e 7DC entre a amostra feminina não é significativa, conforme resultado do Teste $Z(Z=-1,31)$. Isso significa que ambos os testes podem ser utilizados, pois o resultado é fidedigno.

fato das mulheres apresentarem um $\% \mathrm{G}$ maior que o dos homens em ambas as equações é devido às distintas necessidades orgânicas de cada gênero, como por exemplo, o tempo gestacional que requer um grande consumo de energia, neste caso a provinda da gordura subcutânea ${ }^{13}$.

Estatisticamente podemos afirmar, com $99 \%$ de confiança, que a diferença existente nos resultados entre ○ \%G masculino e o \%G feminino do Teste Jackson \& Pollock de 3DC é significante, conforme resultado do Teste $Z(Z=-5,22)$, e no de 7DC, conforme resultado do Teste $Z(Z=-7,36)$.

O fato de uma porcentagem maior da amostra feminina, em relação à porcentagem da amostra masculina, não apresentar excesso de gordura, não quer dizer que as mulheres apresentaram menos gordura subcutânea do que os homens. Elas até podem ter menos gordura, mas a classificação de seu $\% G$ é diferente.

Este excesso de gordura em uma porcentagem da amostra, no caso da comparação entre gêneros na equação de 7DC, pode ser um reflexo da mudança da prática alimentar e de atividade física que esta acontecendo atualmente, onde se ingere uma quantidade muito alta de alimentos ricos em gordura e são realizadas atividades físicas com pouca frequência.
Esta variação entre as equações pode ser explicada em função dos diferentes locais e quantidades de DC aferidas, lembrando que a distribuição da gordura não é homogênea no corpo humano.

\section{REFERÊNCIAS}

1. Queiroga MR. Avaliação da composição corporal. Em: Testes e medidas para avaliação da aptidão física relacionada a saúde em adultos. Rio de Janeiro: Guanabara Koogan; 2005.

2. Pitanga FJG. Testes, medidas e avaliação em educação física e esportes. $3^{a}$ ed. São Paulo: Phorte; 2004

3. Castro L. Medidas e avaliação em educação física. Porto Alegre: Pontifícia Universidade Católica do Rio Grande do Sul; 2006.

4. Heyward VH, Stolarczyk LM. Fundamentos da composição corporal. Em: Avaliação da composição corporal aplicada. São Paulo: Manole; 2000.

5. Heyward VH. Avaliação da composição corporal. Em: Avaliação física e prescrição de exercício: técnicas avançadas. $4^{a}$ ed. Porto Alegre: Artmed; 2004.

6. Tristschler KA. Fundamentos em avaliação. Em: Medidas e avaliação em educação física e esportes. $5^{a}$ ed. São Paulo: Manole; 2003.

7. Fernandes Filho J. Avaliação antropométrica. Em: A prática da avaliação física: testes, medidas e avaliação física em escolares, atletas e academias de ginásticas. $2^{a}$ ed. Rio de Janeiro: Shape; 2003.

8. Costa RF. Conhecendo a composição corporal. Em: Composição corporal: teoria e prática da avaliação. São Paulo: Manole; 2001.

9. Martins JCB, Giannichi RS. Antropometria. Em: Avaliação \& prescrição de atividade física: guia prático. $3^{a}$ ed. Rio de Janeiro: Shape; 2003.

10. Nahas MV. Atividade física, saúde e qualidade de vida. $3^{a}$ ed. Londrina: Midiograf; 2003

11. Triola M. Introdução à estatística. $9^{a}$ ed. Rio de Janeiro: LTC; 2005.

12. Farias ES, Salvador MRD. Antropometria, avaliação corporal e atividade física escolar. Rev Bras Cineantropom Desempenho Hum. 2005;7(7):21-9.

13. Nunes VGS. Prescrição de atividades físicas para pessoas normais e com problemas especiais. Pelotas: Princesa; 1999.

Recebido: 02/12/08 - Aceito: 13/03/2009 